\title{
Apparent metabolisable energy and ileal crude protein digestibility of various treated palm kernel cake based diets for heat-stressed broiler chickens
}

\begin{abstract}
A digestibility trial was conducted to determine the apparent metabolisable energy (AME) and apparent ileal digestibility (AID) of crude protein in broilers fed various treated palm kernel cake (PKC) based diets under unheated and heated conditions. A total of 240 day-old female broiler chicks (Cobb 500) were randomly distributed to 60 battery cages in environmentally controlled rooms and fed standard broiler diets from day 1 to 28 . From day 29 to 35, chickens were assigned to one of the following 6 diets: (1) 25\% untreated PKC, (2) $25 \%$ shell-less PKC (SPKC), (3) 25\% enzyme treated PKC (EPKC), (4) 25\% extruded PKC (XPKC), (5) 25\% extruded shell-less PKC (XSPKC) or (6) 0\% PKC (control). From day 2935 , equal numbers of birds from each diet were exposed to either $24 \pm 1{ }^{\circ} \mathrm{C}$ (unheated) or $34 \pm$ $1^{\circ} \mathrm{C}$ for $6 \mathrm{~h}$ daily (heated). No significant diet $\times$ temperature interactions were observed for AME or AID of CP. Regardless of temperature, XPKC, XSPKC, SPKC and EPKC significantly increased the AME values by 16, 29, 31 and $35 \%$, respectively compared to untreated PKC. In comparison to untreated PKC, the AID of $\mathrm{CP}$ was also significantly increased in EPKC (33\%), SPKC (29\%) and XSPKC (26\%) but not in XPKC. Irrespective of diet, heat-stressed birds had significantly lower AME and AID of CP compared to the unheated group. These results showed that enzyme supplementation and shell removal treatments improved both AME and AID of CP of the PKC. However, extrusion improved AME but not AID of CP.
\end{abstract}

Keyword: Broiler; Nutrition; Heat stress; Palm kernel cake; Extrusion; Enzyme supplementation; Nutrient digestibility 